\title{
SED1 Gene Length and Sequence Polymorphisms in Feral Strains of Saccharomyces cerevisiae
}

\author{
Ilaria Mannazzu, ${ }^{*}$ Emanuela Simonetti, Paola Marinangeli, Emanuela Guerra, $\uparrow$ \\ Marilena Budroni, $\$$ Madan Thangavelu, and Francesca Clementi \\ Dipartimento di Biotecnologie Agrarie ed Ambientali, Università di Ancona, Ancona, Italy
}

Received 13 May 2002/Accepted 2 August 2002

\begin{abstract}
The SED1 gene (YDR077W), coding for the major cell wall glycoprotein of Saccharomyces cerevisiae stationary-phase cells, contains two blocks of tandem repeat units located within two distinct regions of the nucleotide sequence. A PCR survey of the SED1 open reading frames (ORFs) of 186 previously uncharacterized grape must isolates of $S$. cerevisiae yielded 13 PCR profiles arising from different combinations of seven SED1 length variants in individuals homozygous or heterozygous for the gene. Comparison of the nucleotide sequences of a group of representatives of each of the seven length variants with those of $\mathrm{S} 288 \mathrm{C}$ and the type strain, CBS1171, unequivocally identified them as SED1 alleles and provided evidence for the presence of two minisatellite-like sequences, variable in length, within the ORF of an $S$. cerevisiae gene. The segregation analyses of the $S E D 1$ length variants and other genetic markers in 13 isolates representative of each PCR profile suggested that molecular mechanisms involved in minisatellite expansion and contraction may be responsible for $S E D 1$ heterozygosities within a population of homothallic must isolates of $S$. cerevisiae.
\end{abstract}

SED1 (YDR077W), initially identified as a multicopy suppressor of the ERD2 deletion (15), has more recently been shown to encode the most abundant cell wall glycoprotein of Saccharomyces cerevisiae stationary-phase cells (28). Sed1p is rich in serines and threonines and, like other cell wall proteins, has $\mathrm{N}$ - and $\mathrm{C}$-terminal hydrophobic domains, multiple sites for glycosylation with both $\mathrm{N}$ - and O-linked sugars, and a signal sequence for the addition of a glycosylphosphatidylinositol anchor at the carboxy terminus $(6,13,14)$. Sed1p is not essential for normal growth $(15,28)$. However, a $S E D 1$ deletion results in decreased resistance to the action of zymolyase with respect to wild-type cells (28). This phenotype is more evident in stationary-phase cells, implicating the involvement of Sed1p in stress resistance during that growth phase. SED1 is strongly up-regulated at the diauxic shift upon glucose depletion (9) and is highly expressed around the $\mathrm{M}$ phase of the cell cycle (7, 29 ) and in the presence of aluminum and zinc (11). The presence of both stress-responsive elements (CCCCT and AGGGG) in the promoter and upstream region of the gene (28) and of three putative PEST regions (8), which may be necessary for rapid changes in concentration of the protein in response to environmental stimuli $(24,25)$, has suggested a role for Sed $1 \mathrm{p}$ in providing resistance to biotic and abiotic stresses.

Analysis of the amino acid sequence of Sed1p in S. cerevisiae S288C revealed the presence of repeated amino acid motifs localized within two distinct regions of the polypeptide chain

\footnotetext{
* Corresponding author. Mailing address: Dipartimento di Biotecnologie Agrarie ed Ambientali, Università degli Studi di Ancona, Via Brecce Bianche 60131, Ancona, Italy. Phone: 31071 2204782. Fax: 31 071 2204858. E-mail: ilaria@popcsi.unian.it.

$\dagger$ Present address: Dipartimento di Biologia Cellulare ed Oncologia, Consorzio M. Negri Sud, Chieti, Italy.

$\ddagger$ Present address: Dipartimento di Scienze Ambientali Agrarie e Biotecnologie Agro-Alimentari, Università degli Studi di Sassari, Sassari, Italy.
}

(15). Based on the SED1 nucleotide sequence of S288C, the first region (region 1) contains three repeat units of $66 \mathrm{bp}$ and a truncated one of $42 \mathrm{bp}$ arranged in the following order: 66, $42,66,66$. The penultimate codon of each 66-bp unit encodes an asparagine residue that is a potential $\mathrm{N}$-linked glycosylation site. The second region (region 2) contains two 153-bp repeats and the information for a total of six cysteine residues. Similar to their role in mucins, the cysteine residues may be involved in one or more functions, such as disulfide-dependent intramolecular interactions required for protein folding or inter-Sed1p interactions on the cell wall, or in interactions with other cell wall proteins, or they may be necessary for higher-order assemblies involving Sed1p.

Our objective in this study was to assess whether the observed tandem repeats could mimic a minisatellite-like behavior and cause length polymorphism in an S. cerevisiae gene coding for a structural protein. Thus, after a preliminary PCR survey of strain collections and previously uncharacterized $S$. cerevisiae isolates from diverse sources (I. Mannazzu and M. Thangavelu, unpublished data), we surveyed SED1 sequence variation in a collection of previously uncharacterized grape must isolates of $S$. cerevisiae and observed abundant SED1 polymorphisms in the population analyzed. In this paper, we report the presence of minisatellite-like sequences within the open reading frame (ORF) of the $S E D 1$ gene and speculate on the reasons for $S E D 1$ length variation and heterozygosis in feral strains of $S$. cerevisiae.

\section{MATERIALS AND METHODS}

Strains. S. cerevisiae S288C and CBS1171 were used as reference strains. CBS1171 was isolated from "brewer's top yeast," Oranjeboom Brewery, Rotterdam, The Netherlands, by A. C. van Wijk in February 1925 and was deposited at the Centraalbureau voor Schimmelcultures, Utrecht, The Netherlands, in the same period. This strain, considered the type strain for $S$. cerevisiae, is also known as ATCC 18824, CCRC 21447, DBVPG 6173, DSM 70449, IFO 10217, IGC 4455, JCM 7255, NCYC 505, and NRRLY-12632 in the respective collections.

The wild $S$. cerevisiae strains were isolated, during the 1998 vintage, from a 
TABLE 1. Origin of isolates and distribution of the 13 SED1 PCR profiles within the population analyzed

\begin{tabular}{|c|c|c|c|c|c|c|c|c|c|c|c|c|c|c|c|}
\hline \multirow{2}{*}{ Cellar } & \multirow{2}{*}{$\begin{array}{l}\text { Vat } \\
\text { no. }\end{array}$} & \multirow{2}{*}{$\begin{array}{c}\text { Grape } \\
\text { must }\end{array}$} & \multicolumn{13}{|c|}{$\begin{array}{l}\text { No. of isolates harboring the observed } \\
\text { SED1 PCR profile }\end{array}$} \\
\hline & & & A & B & $\mathrm{C}$ & $\mathrm{D}$ & $\mathrm{E}$ & $\mathrm{F}$ & G & $\mathrm{H}$ & I & $\mathrm{L}$ & M & $\mathrm{N}$ & $\mathrm{O}$ \\
\hline \multirow[t]{4}{*}{1} & 1 & Pecorino & & 4 & & 17 & 2 & & & & 8 & & & & 3 \\
\hline & 2 & Sangiovese & & & & 9 & & & & 2 & 7 & & & & 1 \\
\hline & 3 & Passerina & & 1 & & 13 & 2 & 1 & & & 5 & & & & \\
\hline & 4 & Montepulciano & 1 & 5 & & 1 & 7 & & 1 & & 2 & & & & 1 \\
\hline \multirow[t]{4}{*}{2} & 5 & Pecorino & & & 1 & 16 & 3 & & & & & & 1 & & \\
\hline & 6 & Sangiovese & & & & 12 & & & & & & & & 1 & \\
\hline & 7 & Passerina & & & & 10 & 5 & & & & 1 & & & & \\
\hline & 8 & Montepulciano & & & 1 & 2 & 4 & & & 14 & & & & & \\
\hline 3 & 9 & Verdicchio & & & & 10 & 6 & & & 1 & 3 & 1 & & & 1 \\
\hline
\end{tabular}

total of nine vats, each containing must of a single grape variety, and sampled in three separate wine cellars located in a traditional wine-producing area of the Italian region of Marche (Table 1). The musts were collected from the fermentation vats immediately after crushing of the grapes and transferred to sterile 250-ml flasks, which were taken to the laboratory within $8 \mathrm{~h}$ after collection and incubated statically at $25^{\circ} \mathrm{C}$. The yeasts were isolated by streaking triplicate must aliquots on WL nutrient agar (Oxoid) immediately upon sampling (time zero) and at different enrichment stages (early, middle, and late) in order to collect the strains acting in sequence during must fermentation. A total of 286 colonies exhibiting Saccharomyces morphology on WL agar were purified by repeated streaking on YEPD, and 186 isolates, confirmed as belonging to the species $S$. cerevisiae, were utilized in the present study. They were identified according to the method of Vaughan-Martini and Martini (31), utilizing the dichotomic key proposed by Boulton et al. (4) as described by Guerra et al. (12).

Media and growth conditions. WL agar was used for yeast isolation, YEPD ( $2 \%$ glucose, $1 \%$ yeast extract, $2 \%$ peptone, $1.8 \%$ agar) was used for yeast cultivation, and $\mathrm{K}$ acetate $(0.1 \%$ glucose, $0.25 \%$ yeast extract, $0.98 \% \mathrm{~K}$ acetate, $2 \%$ agar) was utilized as a sporulation medium. The media for yeast identification were as described by Guerra et al. (12). The ability to ferment galactose (GAL), melibiose (MEL), trehalose (TRE), maltose (MAL), cellobiose (CEL), sucrose (SUC), and raffinose (RAF) was tested on solid medium (1\% yeast extract, $2 \%$ peptone, $2 \%$ agar) supplemented with the corresponding sugar plus the $\mathrm{pH}$ indicator bromothymol blue and adjusted to $\mathrm{pH} 7.5$ after being autoclaved, as described by Mortimer et al. (21). The ability to assimilate nonfermentable carbon sources was assessed on GLY medium (1\% yeast extract, $2 \%$ peptone, $3 \%$ glycerol, $1 \%$ ethanol [added after autoclaving], $2 \%$ agar). Copper resistance was tested as the ability to grow on CUP medium ( $0.7 \%$ yeast nitrogen base, $2 \%$ glucose, $2 \%$ agar, supplemented with $60 \mathrm{mg}$ of copper sulfate liter $^{-1}$ ). Hydrogen sulfide production was assayed on the basis of the color of the colonies (cream or brown) on Biggy agar (Difco). Unless otherwise stated, liquid cultures were incubated at $28^{\circ} \mathrm{C}$ with shaking $(200 \mathrm{rpm})$.

DNA extraction, PCR conditions, and restriction analyses. Total genomic DNA for PCR analysis was isolated from 24-h cultures as described by Ushinsky et al. (30). The PCR primers SED1FOR (5'-ATGAAATTATCAACTGTCCT ATTATCTGCCGG-3'; bases 1 to 32) and SED1REV (5'-TTATAAGAATAA CATAGCAACACCAGCCAAACC-3'; bases 1017 to 984) were designed on the S. cerevisiae S288C sequence. PCR amplification reactions were performed on a Perkin-Elmer Gene AMP PCR System 9700 in 25- $\mu$ l reaction mixtures containing $2 \mu \mathrm{l}$ of template DNA (approximately $10 \mathrm{ng} / \mu \mathrm{l}$ ), $0.5 \mathrm{U}$ of DyNAzyne II DNA polymerase (Finnzymes), $1 \times$ reaction buffer $\left(\mathrm{Mg}^{2+}\right.$ free), $1.5 \mathrm{mM} \mathrm{MgCl}$, $100 \mu \mathrm{M}$ each deoxynucleoside triphosphate, and 2 pmol each of SED1FOR and SED1REV primers. The reactions were run for 35 cycles as follows: denaturation at $94^{\circ} \mathrm{C}$ for $1 \mathrm{~min}$, annealing at $64^{\circ} \mathrm{C}$ for $1 \mathrm{~min}$, and elongation at $72^{\circ} \mathrm{C}$ for $2 \mathrm{~min}$. An initial denaturation step at $94^{\circ} \mathrm{C}$ for $3 \mathrm{~min}$ and a final $7-$ min extension at $72^{\circ} \mathrm{C}$ were performed. The PCR products were analyzed by electrophoresis on a $1.4 \%$ agarose gel in $1 \times$ Tris-borate-EDTA buffer. The gel images were visualized by means of a Bio-Rad Gel DOC 1000 and acquired with Multi-Analyst software (Bio-Rad). The restriction fragment length polymorphism (RFLP) analysis of the SED1 amplicons was performed as follows: $25 \mu \mathrm{l}$ of the PCR product was digested overnight with an excess of $\mathrm{HpaII}$ and KpnI in a final volume of $50 \mu \mathrm{l}$. The restriction fragments were analyzed as described above.

DNA sequencing and sequence analyses. PCR products were purified using a Microcon-PCR Centrifugal Filter Devices (Millipore) PCR template purification kit or by means of the QIAquick gel extraction kit protocol (Qiagen) and sequenced directly. Sequencing reactions were prepared by PCR by using SED1FOR and SED1REV and the internal primers S1 (5'-TTACAACCCATC TACTGACTACACC-3') and S2 (5'-GGTGTAGTCAGTAGATGGGTTGTA A-3') with the ABI PRISM Big Dye Terminator Cycle Sequencing Ready Reaction kit (Perkin-Elmer). The products of these reactions were purified on Centri-Sep spin columns (Amicon), dried, denatured by adding Template Suppression Reagent (Perkin-Elmer), and sequenced by using the ABI PRISM 310 Genetic Analyzer (Perkin-Elmer). The primary sequence alignment was performed using the Multiple Alignment Program (http://dot.imgen.bcm.tmc.edu: 9331). Minor adjustments of the output alignment were performed by hand.

Tetrad analysis. Yeasts grown on YEPD were streaked on sporulation medium and incubated at $25^{\circ} \mathrm{C}$ until asci were observed microscopically. Depending on spore viability, a total of 16 to 48 asci for each isolate were dissected as described by Rose et al. (26). The single-spore progeny from asci with four viable meiotic products were grown on YEPD and replica plated onto GAL, MEL, TRE, MAL, CEL, SUC, RAF, Biggy agar, CUP, and GLY as described by Mortimer et al. (21). Sporulation efficiency was determined as the sum of fourand three-spore asci divided by the number of asci dissected. Spore viability was determined as the number of viable spores divided by the total number of spores dissected (5). Homothallism was assessed by examining microscopically the abilities of the meiotic derivatives from an ascus to sporulate. Segregation of SED1 alleles among single-spore progeny was analyzed by PCR.

Nucleotide sequence accession numbers. The sequences of the CBS1171 SED1 gene and of Sed1-1 to Sed1-7 were deposited in GenBank under accession numbers AF510219, AF510220, AF510221, AF510222, AF510223, AF510224, AF510225, and AF510226, respectively.

\section{RESULTS}

SED1 polymorphisms in grape must isolates of $S$. cerevisiae. The indication that the $S$. cerevisiae SED1 coding region is variable in length was based on PCR amplification of the SED1 ORFs of a small and random selection of laboratory, food, and industrial strains isolated from diverse sources (Mannazzu and Thangavelu, unpublished data). These preliminary experiments also suggested that a simple PCR assay could be used to analyze SED1 variation in a large number of isolates. We therefore decided to study SED1 length polymorphism in a collection of wine yeasts made up of $186 \mathrm{~S}$. cerevisiae isolates collected in 1998 from fermenting musts. These yeasts were isolated as part of a study aiming to characterize the autochthonous strains associated with traditional wine fermentation in the Marche region of Italy and, coming from musts of different grape varieties sampled from nine vats located in three different cellars, were thought to provide a good source of biodiversity.

Under relatively stringent PCR conditions, the primer pair based on the $S$. cerevisiae S288C SED1 sequence was utilized to amplify total DNA from the two reference strains, CBS1171 and S288C, and from all the must isolates. Both of the reference strains produced a single amplicon; S288C yielded the expected 1,017-bp product, while CBS1171 yielded a product which was smaller than that of S288C (Fig. 1). The $186 \mathrm{~S}$. cerevisiae must isolates displayed striking differences in the electrophoretic profiles of their PCR products. The major proportion of the isolates $(72 \%)$ yielded a single amplification product of variable length, suggesting either a single copy of the gene per haploid genome or multiple copies of the same size in a diploid or polyploid genome. The remaining isolates contained two or three SED1 amplicons of different lengths per genome complement. The SED1 amplicons ranged from approximately 950 to $1,300 \mathrm{bp}$.

Based on the observed SED1 PCR profiles, the 186 must isolates could be grouped into 13 classes designated $\mathrm{A}$ to $\mathrm{O}$ 


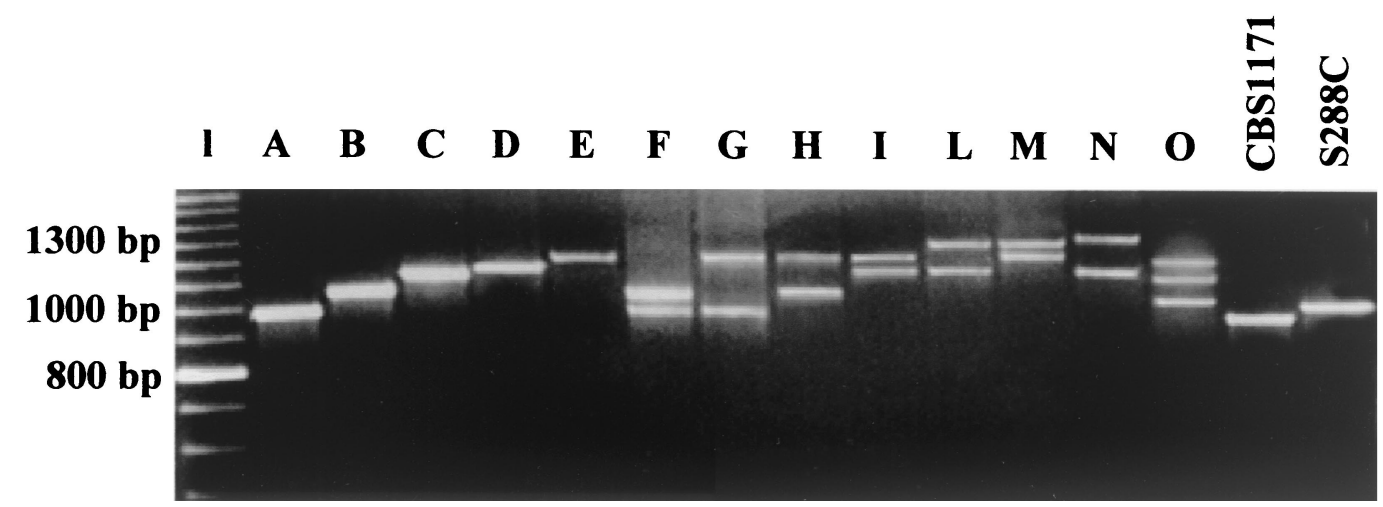

FIG. 1. SED1 gene polymorphisms. PCR primers designed on the $S E D 1$ sequence were utilized to amplify the $S E D 1$ genes of the two reference strains, CBS1171 and S288C, and of the 186 must isolates of S. cerevisiae. Lane 1, 100-bp ladder (Amersham-Pharmacia); lanes A to O, PCR profiles observed within the population analyzed.

(Fig. 1). Interestingly, while the PCR profile and SED1 amplicon size of the type strain, CBS1171, were similar to the class A PCR profile, the 1,017-bp PCR product of S288C did not correspond to any of the PCR profiles observed in the population. Moreover, it was interesting that the SED1 PCR profile $\mathrm{D}$ occurred in all the vats sampled, while some of the others, such as A, F, G, L, M, and N, occurred very rarely and were exclusive to some of the vats (Table 1).

RFLP analysis of SED1 amplicons. Based on the restriction map of the SED1 ORF of S288C (http://genome-www.stanford .edu/Saccharomyces/), it was easy to observe that the HpaII and $K p n I$ restriction enzymes could be used as convenient markers to localize the regions of the gene involved in the observed length variations. In fact, when digested with HpaII, the SED1 ORF of S288C yields two well-resolved fragments. The shorter one (333 bp) contains all of region 1, including the mosaic of 66- and 42-bp repeat units, while the larger fragment (654 bp) contains region 2, which includes the two 153-bp repeats. KpnI cuts the $S E D 1$ gene within region 1 and yields a band corresponding to the 42-bp block located within this region (data not shown). Comparison of the HpaII and KpnI restriction products of the S288C and CBS1171 SED1 genes readily localized the observed length difference to region 1 . In fact, the S288C region 1 fragment was about 40 bp longer than the corresponding fragment in CBS1171, possibly due to the lack of the 42-bp block in the latter (data not shown). Similarly, the HpaII and KpnI restriction profiles of the SED1 amplification products of the isolates presenting PCR profiles $\mathrm{A}$ to $\mathrm{O}$ revealed that the observed length polymorphisms were due to length variation localized in region 1 and/or region 2 . The restriction analyses of the $S E D 1$ amplicons also showed that all the PCR profiles could invariably be considered as derived from combinations of seven SED1 length variants labeled Sed1-1 to Sed1-7, from the shortest to the longest (Fig. 2). Interestingly, the SED1 amplicons of the must isolates never contained the short DNA fragment including the 42-bp block present in S288C and were in that respect similar to that in CBS1171 (Fig. 2).

Sequence analyses of the SED1 length variants. In order to refine the results of the restriction analyses and obtain a detailed structure of the coding region of the gene, the SED1 ORFs of the $S$. cerevisiae type strain, CBS1171, and a repre- sentative of each of the seven SED1 length variants were sequenced.

Consistent with the restriction analyses, the SED1 sequences of S288C and CBS1171 differed by a 42-nucleotide sequence in region 1. Based on the predicted amino acid sequence, this short fragment is most likely the result of an in-frame deletion of a duplication of the DNA sequence containing the first 66-bp blocks of region 1 (Fig. 3). The comparison of the nucleotide sequences of the S288C and CBS1171 SED1 genes also revealed the existence of six single-nucleotide polymorphisms (SNPs) located in region 2 of the S288C gene (at positions $459,510,579,630,732$, and 891). All these changes were at the third position of the respective codons and resulted in silent point mutations.

Interestingly, comparison of the nucleotide and amino acid sequences of the seven alleles with those of CBS1171 and S288C revealed that the CBS1171 SED1 ORF was identical to the Sed1-1 allele. Moreover, the 42-bp block in region 1 and the six SNPs in region 2 were unique to S288C.

The Sed1-1 to Sed1-7 ORFs varied in length from 972 to 1,278 bp. Multiple sequence alignment confirmed that the SED1 length polymorphisms were due to the insertion or deletion of perfect duplicates of DNA sequences containing the 66- and 153-bp units in regions 1 and 2 and corresponding to tandem repeats of 22 and 51 amino acids, respectively. Thus, two minisatellite-like DNA sequences that exhibit the property of possessing a variable number of tandem repeats are responsible for SED1 polymorphism in a population of wild yeast (Fig. 4).

The nucleotide sequence alignment revealed the existence of SNPs in the alleles Sed1-2, Sed1-3, Sed1-5, and Sed1-6, all positioned within region 1 (Fig. 2). Comparison of the amino acid sequences predicted on the basis of the nucleotide sequences revealed that all the single-nucleotide polymorphisms occurring at the third position of the codons are silent. On the other hand, the nucleotide substitutions observed at positions 268 in Sed1-2 and 290 in Sed1-2 and Sed1-5 cause the replacement of a threonine with a serine and a leucine with an isoleucine, respectively, in the resulting amino acid sequences (Fig. 2 and 3).

The analysis of the changes in the sequences and the numbers of repeat units within regions 1 and/or 2 provided a way of 


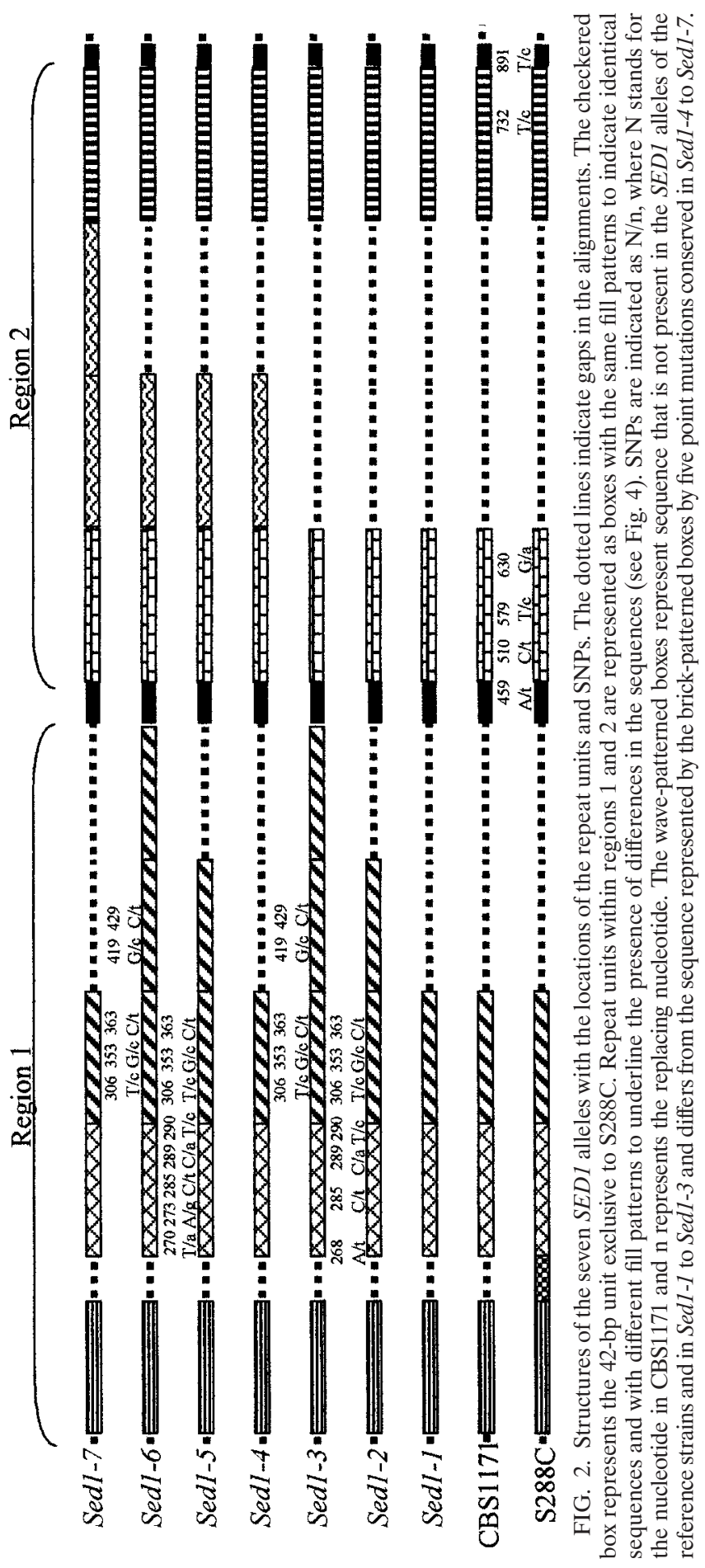

exploring the routes leading to the SED1 polymorphism observed in the must isolates and led us to hypothesize that all the alleles have arisen from a common ancestor as a consequence of tandem duplication events of the repeat units within these two regions. For example, the comparison of both the sequences and structures of Sed1-1, Sed1-4, and Sed1-7 revealed that these three alleles differ in the number of 153-bp repeat units in region 2 (Fig. 2). Moreover, while their first and last 153-bp units are identical to the first and last such units of every other allele sequenced, the two central 153-bp repeat units of Sed1-7 are identical to the central repeat unit of Sed1-4 and differ by five substitutions from the first 153-bp repeat unit of region 2. These observations suggested that Sed1-4 might have originated from Sed1-1 subsequent to a tandem duplication of a 153-bp unit in region 2 followed by the occurrence of the five point mutations in this unit. Similarly, a plausible course of events in the history of Sed1-7 would have involved a tandem duplication of the Sed1-4 central 153-bp unit.

Segregation analysis of the seven SED1 alleles and other genetic markers. Thirteen isolates representative of each PCR profile were subjected to genetic analyses as previously done by Mortimer et al. (21). As shown in Table 2, the 13 isolates were homothallic and exhibited various sporulation efficiencies and spore viabilities, as expected in wine strains of $S$. cerevisiae, due to their complex genetic constitution $(2,8)$. Apart from isolate Sc157, presenting PCR profile A, which was homozygous for all the characteristics considered, all the other isolates presented one or more heterozygosities, as expected in diploid strains (21).

The segregation of the SED1 length variants in the $\mathrm{F}_{1}$ progeny was analyzed by PCR on the monosporial cultures deriving from the four-viable-spore asci generated by each strain. The isolates presenting PCR profiles A to E showed a 4:0 segregation ratio (SED1 amplicons of the same size in each monosporial culture from the same ascus) consistent with the presence of two copies of the same SED1 length variant in a diploid background. Conversely, and as expected in diploid strains heterozygous for the $S E D 1$ gene, isolates presenting PCR profiles F, G, I, L, M, and N showed a 2:2 segregation ratio for the SED1 length variants. Isolates Sc59 and Sc93, presenting PCR profiles $\mathrm{H}$ and $\mathrm{O}$, showed $S E D 1$ segregation ratios which were consistent with the hypothesis of heterozygosity of this gene in a polyploid or polysomic background (Fig. 5), not unusual in wine strains (22).

\section{DISCUSSION}

By using a PCR approach, we have provided evidence for the existence of $S E D 1$ gene polymorphism in a collection of grape must isolates of $S$. cerevisiae. The PCR screen and the PCR-RFLP procedure utilized proved to be effective tools for the analysis of this polymorphism and indicated that the 13 SED1 PCR profiles observed within the collection of must isolates and labeled $\mathrm{A}$ to $\mathrm{O}$ were the result of different combinations of seven SED1 length variants in individuals homozygous or heterozygous for this gene.

The sequence analysis of a representative of each of the observed SED1 length variants unequivocally identified them as seven alleles of the $S E D 1$ gene. However, as other alleles of the same size but with meaningful SNPs could have gone 
S28 80 CBS 1171 Sed $1-7$ $S \in d 1-6$ $S \in d 1-5$ sed $1-4$ Sed $1-3$

$S \in d 1-2$

Sed 1-1

S $288 \mathrm{C}$ CBS 1171

Sed 1-7

sed $1-6$

Sed $1-5$

Se d $1-4$

Sed $1-3$

Sed $1-2$

Sed $1-1$

S $288 \mathrm{C}$

CBS1171

Sed $1-7$

Sed $1-6$

Sed $1-5$

sed $1-4$

Se d $1-3$

Sed $1-2$

Sed $1-1$

$5288 \mathrm{C}$

CBS 1171

$S$ ed $1-7$

Sed $1-6$

Sed $1-5$

Se d $1-4$

$S$ ed $1-3$

Sed $1-2$

sed 1-1

$5288 \mathrm{C}$

CBS 1171

Sed $1-7$

sed $1-6$

Sed $1-5$

$S$ ed $1-4$

Sed $1-3$

Sed $1-2$

sed $1-1$

52880

CBS 1171

Sed $1-7$

Sed $1-6$

Sed $1-5$

Sed $I-4$

Sed $1-3$

Sed $1-2$

Sed $1-1$

S $28 B C$

C BS 1171

Sed 1 - 7

Sed $1-6$

$S \in d 1-5$

Se d 1-4

Sed $1-3$

sed $1-2$

sed $1-1$

$5288 \mathrm{C}$

CBS1171

Sed $1-7$

Sed $1-6$

Sed $1-5$

Sed $I-4$

Sed I- 3

sed $1-2$

sed $1-1$

$5288 \mathrm{C}$

C BS 1171

Sed $1-7$

sed $1-6$

sed $1-5$

Sed $1-4$

sed I- 3

sedi- 2

sed $1-1$

MKLSTVLLSAGLAST TLAQESNSTSASSTDVTSSSSISTSSGSVTITSSEAPESDNGTST

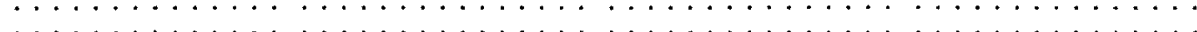

60

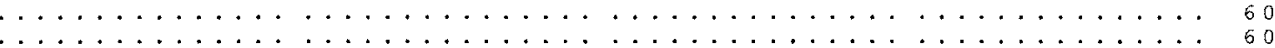

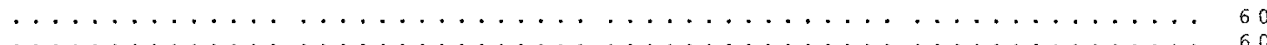

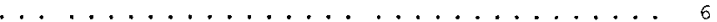
AAPTETSTEAPTTAI PTNGTSTEAPTTAIP TNG Tำ $\cdots \cdots \cdots$

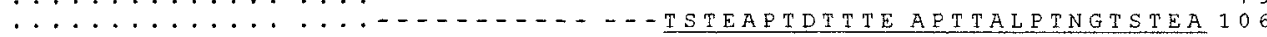
$\ldots \ldots \ldots \ldots \ldots \ldots \ldots \ldots \ldots \ldots$

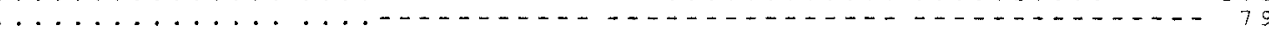

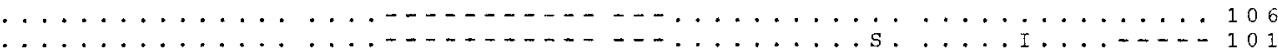

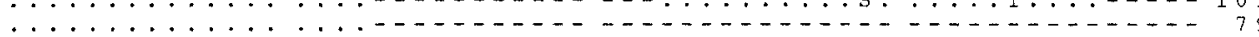
-

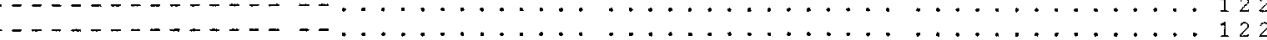

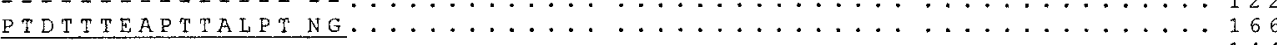

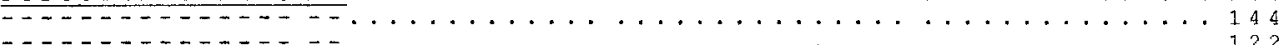

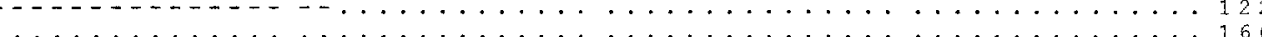

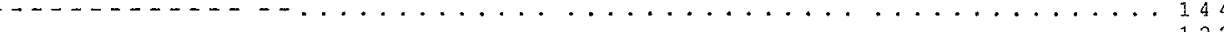

GTTSAEPTTSLFES NTTTPEYN PSTDYT TDYTVVTEYTTYCPEPTTETTNGKTYTVTE 196

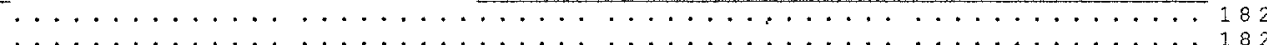

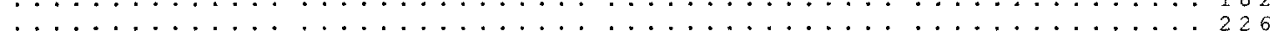

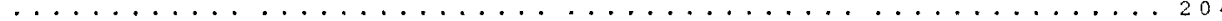

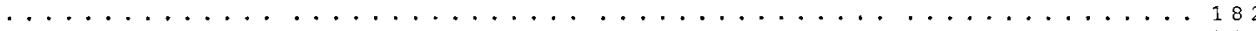
$\ldots \ldots$ PTTLTIT TICPCTIEK PILIITDCPCTIEK $\ldots \ldots$. . . . . . . TTSTTEYTVVTEYTTYCPEPTTETTNGKTYTVTEPTTLTITDC 242

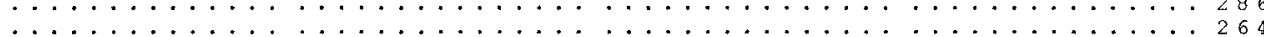
$\ldots \ldots \ldots \ldots \ldots \ldots \ldots \ldots \ldots \ldots \ldots$

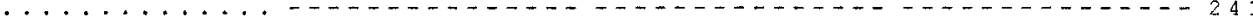

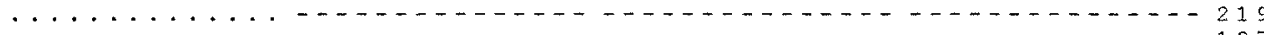

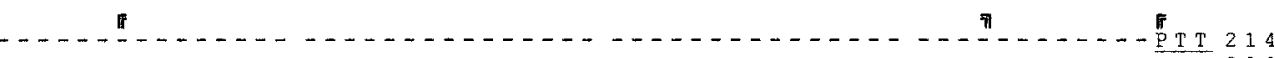
PCTIEKPTTTSTTEY TVVTEYTTYCPEPTT ETTNGKTYTVTEPTT LTITDCPCTIEK. . . 302 $\ldots \ldots . \ldots \ldots$

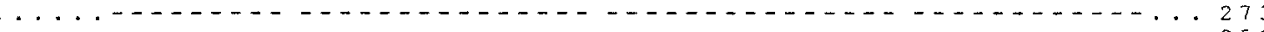
-

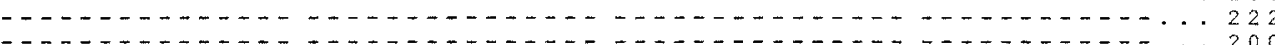
TSTIEYTVVTEYTTY CPEPTTETTNGKTYT VTEPTTLTITDCPCTIEKSEAPESSVPVTE 274

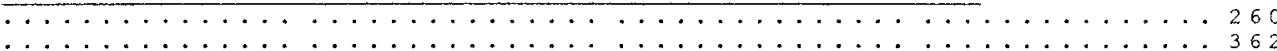

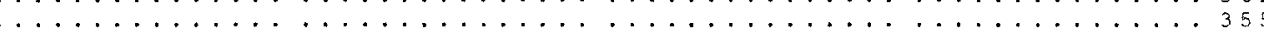

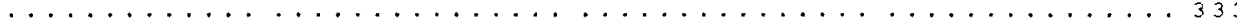

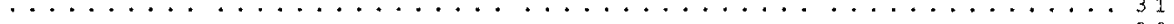

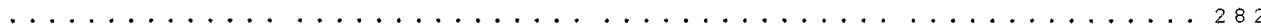

SKGTTTKETGVTTKQTTANPSLTVSTVVPVSSSASSHSVVINSNG ANVVVPGALGLAGVA 334

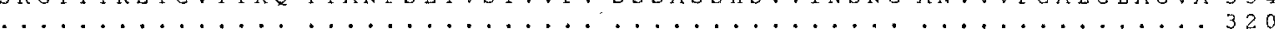

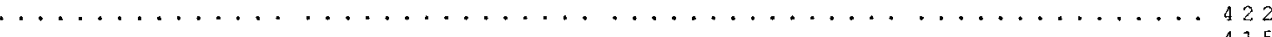
$\ldots \ldots \ldots \ldots$

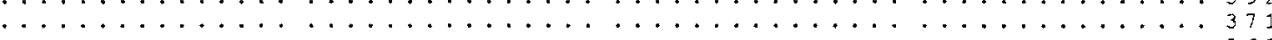

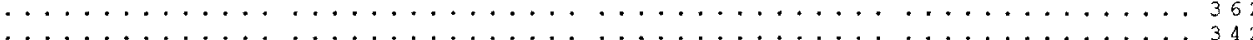

MLEL 338

... 324

$\cdots 426$

... 419

(

$\ldots 368$

.. 34

FIG. 3. Multiple alignments of the predicted amino acid sequences of the seven SED1 alleles and the SED1 genes of CBS1171 and S288C. The repeat units within regions 1 and 2 are underlined. The dashes indicate gaps introduced to maximize the alignment, and the dots indicate identical residues. Amino acid coordinates are provided. The beginning and end of each repeat unit is indicated by a left and right "corner," respectively, above the sequence. 
Region 1

172

ACC

238

ACC

304

ACT TCT ACT GAAGCT CCAACT GATACTACTACTGAAGCTCCAACCACCGGTCTTCCAACCAACGG'

Region 2

610

ACCACT GAC TACACC GTA GTCACT GAA TAT ACT ACT TAC TGT CCA GAA CCAACCACT TTCACC ACAAAC

916

ACC ACC GAA TACACT GTA GTCACT GAGTACACT ACT TAC TGT CCA GAACCAACCACT TTCACCACAAAC

679

747

GGT AAGACT TACACT GTCACT GAACCAACCACT TTGACT ATCACT GACTGT CCA TGT ACT ATT GAAAAG

985

1053

GGT AAGACT TACACT GTCACT GAACCAACCACATTGACT ATCACT GACTGTCCA TGCACCATT GAGAAG
Repeat 1

Repeat 2

Repeat 3

Repeat 1

Repeat 2

Repeat 1

Repeat 2

FIG. 4. Alignment between repeat units in regions 1 and 2 of CBS1171 SED1 gene. Nucleotide coordinates are given.

undetected by the PCR-RFLP procedure, it is plausible that a higher number of alleles is present within the population analyzed.

Aiming to redefine the structure of the gene and elucidate the sequence of events that may have led to the observed length variations, we first compared the $S E D 1$ sequences of the seven alleles with those of the two reference strains. The results obtained showed that the seven alleles analyzed have high

TABLE 2. Genetic analyses of 13 isolates representative of SED1 PCR profiles

\begin{tabular}{|c|c|c|c|c|c|c|c|c|c|c|c|c|c|}
\hline \multirow{2}{*}{ Characteristic $^{a}$} & \multicolumn{13}{|c|}{ Result for strain ${ }^{b}$ : } \\
\hline & Sc157 & Sc205 & $\operatorname{Sc} 23$ & Sc42 & Sc44 & Sc200 & Sc151 & Sc59 & Sc28 & Sc112 & Sc12 & Sc143 & Sc93 \\
\hline Class & A & B & $\mathrm{C}$ & $\mathrm{D}$ & $\mathrm{E}$ & $\mathrm{F}$ & G & $\mathrm{H}$ & I & $\mathrm{L}$ & M & $\mathrm{N}$ & $\mathrm{O}$ \\
\hline Total no. of asci & 16 & 16 & 16 & 16 & 16 & 16 & 16 & 32 & 16 & 16 & 16 & 16 & 48 \\
\hline \multicolumn{14}{|l|}{ Via. spore/ascus } \\
\hline 4 & 1 & 15 & 13 & 3 & 8 & 13 & 3 & 5 & 1 & 3 & 2 & 15 & 7 \\
\hline 3 & 1 & 0 & 0 & 4 & 0 & 0 & 2 & 8 & 8 & 9 & 3 & 0 & 16 \\
\hline 2 & 3 & 0 & 1 & 6 & 8 & 0 & 6 & 10 & 1 & 4 & 4 & 0 & 9 \\
\hline 1 & 4 & 0 & 0 & 0 & 0 & 0 & 3 & 3 & 3 & 0 & 3 & 0 & 0 \\
\hline 0 & 7 & 1 & 2 & 3 & 0 & 3 & 2 & 6 & 3 & 0 & 4 & 1 & 16 \\
\hline Spo. eff. & 0.13 & 0.94 & 0.81 & 0.44 & 0.5 & 0.81 & 0.31 & 0.41 & 0.56 & 0.75 & 0.31 & 0.94 & 0.48 \\
\hline Via $\%$ & 27 & 94 & 84 & 56 & 75 & 81 & 52 & 52 & 52 & 73 & 44 & 94 & 49 \\
\hline SPO & $+/+$ & $+/+$ & $+/+$ & $+/+$ & $+/+$ & $+/+$ & $+/+$ & $+/+$ & $+/+$ & $+/+$ & $+/+$ & $+/+$ & $+/+$ \\
\hline GAL & $-1-$ & $+/+$ & $+/+$ & $+/-$ & $+1-$ & $+1-$ & $+/+$ & $+1-$ & $+1-$ & $+1-$ & $-1-$ & $-1-$ & $+1-$ \\
\hline MEL & $-1-$ & $-1-$ & $-1-$ & $-1-$ & $-1-$ & $-1-$ & $-1-$ & $-1-$ & $-1-$ & $-1-$ & $-1-$ & $-1-$ & $-1-$ \\
\hline TRE & $-1-$ & $-1-$ & $-1-$ & $-1-$ & $-1-$ & $-1-$ & $-1-$ & $-1-$ & $-1-$ & $-1-$ & $-1-$ & $-1-$ & $-1-$ \\
\hline MAL & $+/+$ & $+1-$ & $-1-$ & $-1-$ & $+1-$ & $+1-$ & $+1-$ & $+1-$ & $-1-$ & $+1-$ & $+/+$ & $-1-$ & $+/-$ \\
\hline CEL & $-1-$ & $-1-$ & $-1-$ & $-1-$ & $-1-$ & $+1-$ & $-1-$ & $-1-$ & $-1-$ & $-1-$ & $-1-$ & $-1-$ & $-1-$ \\
\hline SUC & $+1+$ & $+1+$ & $+/+$ & $+1+$ & $+1-$ & $+/+$ & $+/+$ & $+/+$ & $+1-$ & $+1-$ & $-1-$ & $+/+$ & $+/+$ \\
\hline RAF & $+/+$ & $+/-$ & $-1-$ & $+/+$ & $-1-$ & $+1-$ & $+/+$ & $+/-$ & $-1-$ & $+/-$ & $-1-$ & $+/-$ & $+/-$ \\
\hline GLY & $+/+$ & $+/-$ & $+/+$ & $+/+$ & $+/+$ & $+/+$ & $+/+$ & $+/+$ & $+/+$ & $+/+$ & $+/+$ & $+/+$ & $+/+$ \\
\hline $\mathrm{H}_{2} \mathrm{~S}$ & $+/+$ & $+/-$ & $+1-$ & $+/+$ & $+1-$ & $+1-$ & $+/+$ & $+1-$ & $+/+$ & $+/+$ & $+/+$ & $+/+$ & $+1-$ \\
\hline $\mathrm{Cu}^{2+}$ & $+/+$ & $+/+$ & $+/+$ & $+/+$ & $+/+$ & $+1+$ & $+/+$ & $+/+$ & $+/+$ & $+/+$ & $+/-$ & $+/+$ & $+/+$ \\
\hline Het & 0 & 4 & 1 & 1 & 4 & 5 & 1 & 4 & 2 & 4 & 1 & 1 & 4 \\
\hline
\end{tabular}

${ }^{a}$ Class refers to the SED1 PCR profile. Total no. of asci, number of four-spore asci dissected; Via. spore/ascus, number of asci with 4, 3, 2, 1, or no viable spores; Spo. eff., sporulation efficiency; Via\%, spore viability; SPO, ability of individual spore clones to sporulate; GAL, MEL, TRE, MAL, CEL, SUC, and RAF, fermentation tests on the corresponding sugar; GLY, glycerol assimilation; $\mathrm{H}_{2} \mathrm{~S}$, hydrogen sulfide production; $\mathrm{Cu}^{2+}$, resistance or sensitivity to copper ions; Het, number of heterozygosities.

${ }^{b}$ The presence of dominant, recessive, or both alleles among spore progeny from tetrads is indicated as $+/+,-/-$, or $+/-$, respectively. 

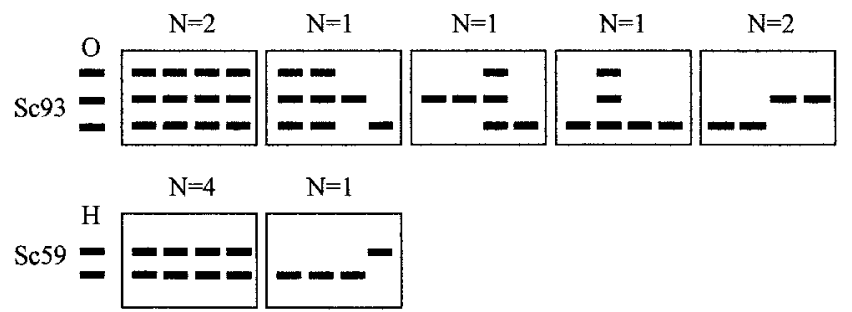

FIG. 5. Segregation of SED1 length variants in $\mathrm{F}_{1}$ progeny. $\mathrm{O}$ and $\mathrm{H}, \mathrm{PCR}$ profiles of isolates $\mathrm{Sc} 93$ and $\mathrm{Sc} 59$, respectively. The boxes contain the SED1 PCR profiles of the monosporial cultures deriving from each ascus. $\mathrm{N}$, number of asci.

structural and nucleotide sequence similarities with the SEDI allele of CBS1171, isolated as brewer's top yeast and considered the type strain of $S$. cerevisiae. On the other hand, the SED1 allele of S288C, which has both a recent "origin" and a complex pedigree (20), differs from those of CBS1171 and all the isolates in both the presence of a 42-bp block in region 1 and six silent SNPs in region 2, which are not present in either CBS1171 or the seven alleles sequenced. Other authors have already highlighted the existence of variations between feral $S$. cerevisiae and S288C, or its isogenic strains, concerning the coding sequences of different genes $(18,19)$. Our data, besides adding another interesting example to this list, underline once again that this laboratory strain may not be considered fully representative of the species $S$. cerevisiae.

The annealing of the SED1 sequences indicates that length variations are due to changes in the number of 66- and 153-bp tandem repeat units which mimic the characteristics of classic minisatellites. Thus, the observed gene length polymorphism is very likely a consequence of the different molecular mechanisms proposed for minisatellite array expansion and contraction (3, 10, 27). Hoyer et al. (16), Andersen and Nilsson-Tillgren (1), and Ingavale et al. (17) have reported the presence of repeat motifs within the ORFs of Saccharomyces carlsbergensis, Candida albicans, and Schizosaccharomyces pombe genes, respectively. Our results, besides suggesting that minisatellites in yeast are not as rare as initially supposed, represent the first description of polymorphic minisatellite-like sequences within the ORF of an S. cerevisiae gene.

Taking into account that Sed1p provides stress resistance during stationary phase, the phase in which $S$. cerevisiae carries out at least two-thirds of the alcoholic fermentation (23), one interesting question would be whether the generation and maintenance of different SED1 alleles offer wine yeasts an adaptive advantage in response to the numerous environmental stresses that they undergo during must fermentation. Indeed, the analyses of the predicted amino acid sequences of the alleles sequenced indicate that the observed changes in the number of the 66-bp repeat units in region 1 may affect the number of potential $\mathrm{N}$-glycosylation sites. This could provide a basis for a great diversity in the sugar-mediated interactions with other cell wall components. Similarly, changes in the number of 153-bp repeat units in region 2, by changing the number of cysteine residues, could have functional and biological consequences for the cell wall properties, in case cysteines are involved in molecular disulfide bonds, or for intermolecular interaction with neighboring Sed1p or other cell wall proteins.
At present, the consequences of the observed length variations for the function of the resulting Sed1p have not been explored. However, the fact that all the different SED1 alleles are transcribed in strains homozygous or heterozygous for this gene (I. Mannazzu and P. Marinangeli, unpublished data) suggests that the observed length polymorphisms are tolerated and that any functional consequence for the resulting proteins should be compatible with the range of functional parameters seen in the population.

Evaluation of the distribution of the 13 SED1 PCR profiles within the population under study showed that the vast majority of the isolates are homozygous for the SED1 gene and indicated that PCR profile D predominates in the population analyzed (Table 1). Interestingly, a PCR survey of the SED1 gene in a population of 132 S. cerevisiae isolates from 26 different grapes and musts sampled in six different cellars located in the Puglia region of Italy showed a similar distribution of SED1 PCR profiles (I. Mannazzu, unpublished data), indicating that these data are probably not the consequence of a population sample bias.

The homothallic behavior, the low number of heterozygosities observed, and the 4:0 segregation ratio of the SED1 alleles in the isolates presenting PCR profiles A to $\mathrm{E}$ are consistent with the "genome renewal" model, according to which diploid homothallic wine strains evolve by meiotic rearrangements which lead to fitter homozygous progeny (21). Also, isolates presenting PCR profiles $\mathrm{F}$ to $\mathrm{O}$, heterozygous for SED1, are homothallic and show a low number of heterozygosities. Thus, based on this evidence, it seems quite unlikely that the coexistence of different $S E D 1$ alleles in these isolates derives from recent conjugation events between strains carrying different alleles. On the other hand, a plausible course of events in the generation of SED1 heterozygosity in feral strains of S. cerevisiae could involve the occurrence of interallelic or intra-allelic recombination in isolates originally homozygous for this gene.

\section{ACKNOWLEDGMENTS}

We are truly grateful to Alan Bakalinsky and Ann Vaughan-Martini for helpful comments on the manuscript.

This work was supported by Fondo di Ateneo, 1999 (I.M.), and by Regione Marche-REG. CEE 2081/83 ob. 5b.

\section{REFERENCES}

1. Andersen, T. H., and T. Nilsson-Tillgren. 1997. A fungal minisatellite. Nature 386: 771 .

2. Bakalinsky, A. T., and R. Snow. 1990. The chromosomal constitution of wine strains of Saccharomyces cerevisiae. Yeast 6:367-382.

3. Bishop, A. J. R., E. J. Louis, and R. H. Borts. 2000. Minisatellite variants generated in yeast meiosis involve DNA removal during gene conversion. Genetics 156:7-20.

4. Boulton, R. B., V. L. Singleton, L. F. Bisson, and R. E. Kunkee. 1996. Yeast and biochemistry of ethanol fermentation, p. 102-192. In R. B. Boulton, V. L. Singleton, L. F. Bisson, and R. E. Kunkee (ed.), Principles and practices of winemaking. Chapman \& Hall, New York, N.Y.

5. Budroni, M., G. Giordano, G. Pinna, and G. A. Farris. 2000. A genetic study of natural flor strains of Saccharomyces cerevisiae isolated during biological ageing from Sardinian wines J. Appl. Microbiol. 89:657-662.

6. Caro, L. H., H. Tettelin, J. H. Vossen, A. F. Ram, H. van den Hende, and F. M. Klis. 1997. In silicio identification of glycosyl-phosphatidylinositolanchored plasma-membrane and cell wall proteins of Saccharomyces cerevisiae. Yeast 13:1477-1489.

7. Caro, L. H., G. J. Smith, P. van Egmond, J. W. Chapman, and M. Klis. 1998. Transcription of multiple cell wall protein-encoding genes in Saccharomyces cerevisiae is differentially regulated during the cell cycle. FEMS Microbiol. Lett. 161:345-349.

8. Coster, F., J. L. Jonniaux, and A. Goffeau. 1995. Analysis of a $32.8 \mathrm{~kb}$ segment of yeast chromosome IV reveals 21 open reading frames including 
TPS2, PPH3, RAD55, SED1, PDC2, AFR1, SSS1, SLU7, and a tRNA for arginine. Yeast 11:673-679.

9. de Risi, J. L., V. R. Iyer, and P. O. Brown. 1997. Exploring the metabolic and genetic control of gene expression on a genomic scale. Science 278:680-686.

10. Doege, K. J., S. N. Coulter, L. M. Meek, K. Maslen, and J. G. Wood. 1997. A human-specific polymorphism in the coding region of the aggrecan gene. J. Biol. Chem. 272:13974-13979.

11. Ezaki, B., R. C. Gardner, Y. Ezaki, H. Kondo, and H. Matsumoto. 1998 Protective roles of two aluminium (Al)-induced genes, HSP150 and SED1 of Saccharomyces cerevisiae, in $\mathrm{Al}$ and oxidative stresses. FEMS Microbiol. Lett. 159:99-105.

12. Guerra, E., G. Sordi, I. Mannazzu, F. Clementi, and F. Fatichenti. 1999 Occurrence of wine yeasts on grapes subjected to different pesticide treatments. Ital. J. Food Sci. 11:221-230.

13. Hamada, K., S. Fukuchi, M. Arisawa, M. Baba, and K. Kitada. 1998. Screening for glycosylphosphatidylinositol (GPI)-dependent cell wall proteins in Saccharomyces cerevisiae. Mol. Gen. Genet. 258:53-59.

14. Hamada, K., H. Terashima, M. Arisawa, and N. Yabuki. 1999. Amino acid residues in the $\omega$-minus region participate in cellular localization of yeas glycosylphosphatidylinositol-attached proteins. J. Bacteriol. 181:3886-3889.

15. Hardwick, K., J. C. Boothroyd, A. D. Rudner, and H. R. Pelham. 1992. Genes that allow yeast cells to grow in the absence of the HDEL receptor. EMBO J. 11:4187-4195.

16. Hoyer, L. L., S. Schere, A. R. Shatzman, and G. P. Livi. 1995. Candida albicans ALS1: domains related to a Saccharomyces cerevisiae sexual agglutinin separated by a repeating motif. Mol. Microbiol. 15:39-54.

17. Ingavale, S. S., R. Kaur, P. Aggarwal, and A. Bachawat. 1998. A minisatellite sequence within the propeptide region of the vacuolar carboxypeptidase $Y$ gene of Schizosaccharomyces pombe. J. Bacteriol. 180:3727-3729.

18. Laizè, V., F. Tacnet, P. Ripoche, and S. Hohmann. 2000. Polymorphism of Saccharomyces cerevisiae acquaporins. Yeast 16:897-903.

19. Liu, H., C. A. Styles, and G. R. Fink. 1996. Saccharomyces cerevisiae S288C has a mutation in FLO8, a gene required for filamentous growth. Genetics 144:967-978.

20. Mortimer, R. K., and J. R. Johnston. 1986. Genealogy of principal strains of the yeast genetic stock center. Genetics 113:35-43.
21. Mortimer, R. K., P. Romano, G. Suzzi, and M. Polsinelli. 1994. Genome renewal: a new phenomenon revealed from a genetic study of 43 strains of Saccharomyces cerevisiae derived from natural fermentation of grape musts. Yeast 10:1543-1552.

22. Puig, S., A. Querol, E. Barrio, and J. E. Pérez-Ortìn. 2000. Mitotic recombination and genetic changes in Saccharomyces cerevisiae during wine fermentation. Appl. Environ. Microbiol. 66:2057-2061.

23. Puig, S., and J. E. Perez-Ortìn. 2000. Stress response and expression pattern in wine fermentations of yeast genes induced at the diauxic shift. Yeast 16:139-148.

24. Rechsteiner, M., and S. W. Rogers. 1996. PEST sequences and regulation by proteolysis. Trends Biochem. Sci. 21:267-271.

25. Rogers, S., R. Wells, and M. Rechsteiner. 1986. Amino acid sequences common to rapidly degraded proteins: the PEST hypothesis. Science 234: 364-368.

26. Rose, M. D., F. Winston, and P. Hieter. 1990. Meiotic mapping, p. 19-29. In M. D. Rose, F. Winston, and P. Hieter (ed.), Methods in yeast genetics-a laboratory course manual. Cold Spring Harbor Laboratory Press, New York, N.Y.

27. Schlotterer, C., and D. Tautz. 1992. Slippage synthesis of simple sequence DNA. Nucleic Acids Res. 20:211-215.

28. Shimoi, H., H. Kitagaki, H. Ohmori, Y. Iimura, and K. Ito. 1998. Sed1p is a major cell wall protein of Saccharomyces cerevisiae in the stationary phase and is involved in lytic enzyme resistance. J. Bacteriol. 180:3381-3387.

29. Spellman, P. T., G. Sherlock, M. Q. Zhang, V. R. Iyer, M. B. Eisen, P. O. Brown, D. Botstein, and B. Futcher. 1998. Comprehensive identification of cell cycle-regulated genes of the yeast Saccharomyces cerevisiae by microarray hybridization. Mol. Biol. Cell 12:3273-3297.

30. Ushinsky, S. C., H. Bussey, A. A. Ahmed, Y. Wang, J. Friesen, B. A. Williams, and R. K. Storms. 1997. Histone H1 in Saccharomyces cerevisiae. Yeast 13:151-161.

31. Vaughan-Martini, A., and A. Martini. 1998. Saccharomyces Meyen ex Rees, p. 358-371. In C. P. Kurtzman and J. W. Fell (ed.), The yeasts, a taxonomic study. Elsevier Press, Amsterdam, The Netherlands. 